\title{
NICE recommends tighter blood sugar control in diabetes to reduce risk of complications
}

On 11 September 2015, we changed some of the wording in the fourth paragraph of this News story (BMJ 2015;351:h4612, doi:10.1136/bmj.h4612), so that our summary of NICE's recommendations was clearer. The last sentence in this paragraph now reads: "adults with newly diagnosed type 1 diabetes should not be offered non-basal-bolus insulin regimens, such as twice daily mixed, basal only, or bolus only." Previously, this read: "adults with newly diagnosed type 1 diabetes should not be offered non-basal-bolus insulin regimens but rather twice daily mixed, basal only, or bolus only."

\section{Cite this as: BMJ 2015;351:h4905}

๑ BMJ Publishing Group Ltd 2015 